\title{
Visual marking: Evidence for inhibition using a probe-dot detection paradigm
}

\author{
DERRICK G. WATSON \\ University of Warwick, Coventry, England \\ and \\ GLYN W. HUMPHREYS \\ University of Birmingham, Birmingham, England
}

\begin{abstract}
Watson and Humphreys $(1997,1998)$ have recently demonstrated that new objects can be prioritized for visual attentional processing by the top-down attentional inhibition of old objects already in the field, a mechanism they called visual marking. The experiments reported here show that the detection of a dim probe dot is impaired when it falls at the location of an old object (Experiments 1 and 3 ) but that this occurs only in conditions in which it is advantageous for subjects to mark (inhibit) old objects (Experiment 2). These results further support previous work showing that visual marking is based on the inhibition of the locations of old objects and that visual marking can be flexibly applied (or withheld), depending on the goals of current behavior.
\end{abstract}

In interactions with the environment, the human visual system is continually confronted with a vast number of objects that fall across the retina. Actions can be addressed to only a few objects at a time (Allport, 1987; Duncan, 1980), so there is a need to prioritize the order in which visual information is processed. In order to behave efficiently, we need to be able to select only the information that is relevant to our current behavioral goals and/or to filter out as much irrelevant information as possible. Theories of visual processing have often emphasized that selection is based on space; for instance, selection operates by increased activation of objects falling in an attended part of the visual field. However, the visual world is not static; changes take place in the spatial positions of objects (when they move or when the observer moves), parts of objects can appear or disappear across time (e.g., because of occlusion), and, on some occasions, prioritization may need to be given to a new event as soon as it occurs. That is, there needs to be selection across time as well as space. Consider, as an example of the need for temporally based selection, the following: When a predator is hunting in a forest, it may have to wait for a prey to emerge against a known background. This ability to prioritize attention to new objects, and to filter out old objects, is of considerable importance for survival.

\section{Attentional Prioritization of New Objects}

Work by Yantis and colleagues (e.g., Yantis, 1993; Yantis \& Hillstrom, 1994; Yantis \& Jonides, 1984) has

This work was supported by a grant from the Medical Research Council (U.K.). Correspondence should be addressed to D. G. Watson, Department of Psychology, University of Warwick, Coventry CV4 7AL, England (e-mail: d.g.watson@warwick.ac.uk). shown that attention can be captured rapidly by new objects. In these studies, Yantis and colleagues used a variant of a visual search task, in which an array of elements is displayed and the observer has to determine whether a prespecified target element is present or not. Initially a number of "camouflaged" letters are displayed (the preview display); these letters have irrelevant line segments that make the letters illegible. After some time (e.g., $1,000 \mathrm{msec}$ ) the camouflage is removed from these old objects, to reveal the identity of the letters; simultaneously a new letter is presented at a previously unoccupied location. Reaction times (RTs) are faster when the target is the new letter than when it is one of the old objects; also, RTs to new-letter targets are unaffected by the number of old objects present. Yantis and Hillstrom showed that this new-object advantage occurred even when the new object was isoluminant with the background (the target being defined by texture, local texture motion, or binocular disparity). They proposed that the advantage was due (at least in part) to new objects capturing attention automatically and that this was not dependent on the occurrence of a luminance increment (cf. Yantis \& Jonides, 1996). Such work suggests that attentional capture by new objects is a relatively passive process that occurs automatically once new events occur.

However, attentional prioritization of new objects may not only occur passively, determined solely by the presentation of a new event; rather, attentional prioritization may depend on an interplay between the salience of changes taking place at old and new (previously unoccupied) locations, and on the attentional deprioritization of old objects. For instance, in the procedure used by Yantis and colleagues, old objects undergo changes in some attributes (e.g., line segments are removed); new objects present an entirely different set of attributes for visual pro- 
cessing. Attentional prioritization may be assigned to new, relative to changed, sets of properties (Yantis \& Jonides, 1996; see also Watson \& Humphreys, 1995). In this last case, the addition of a new property to an old object may make it attentionally salient.

Watson and Humphreys (1997) used a procedure somewhat different from that of Yantis and his colleagues. They employed a variant of a standard color-form conjunction search task (cf. Treisman \& Gelade, 1980). Usually, in such a task, subjects search for a target defined by a conjunction of its color and form (e.g., a blue $\mathrm{H}$ target among green $\mathrm{H}$ and blue $\mathrm{A}$ distractors). This task can be relatively difficult and RTs are linearly related to the number of distractors present with the search slopes on targetpresent trials being around $25-30 \mathrm{msec} / \mathrm{item}$. Such results have been taken to indicate that the search for a colorform conjunction target is spatially serial (Treisman \& Gelade, 1980; but see Humphreys \& Müller, 1993; Townsend, 1972). In contrast to this result, search for a target defined relative to the distractors by its color or form alone can be relatively unaffected by the number of distractors present. Such a result suggests that search for targets defined by a single feature relative to distractors is spatially parallel.

Watson and Humphreys (1997) compared a singlefeature task (search for a blue $\mathrm{H}$ target among blue A distractors) and a conjunction task (search for a blue $\mathrm{H}$ target among green $\mathrm{H}$ and blue A distractors) with a third, gap condition. The gap condition was essentially the same as the conjunction condition except that one set of the distractors (the green $\mathrm{Hs}$ ) was presented for $1,000 \mathrm{msec}$ before the remaining blue items (which would contain the blue $\mathrm{H}$ target when present) were added. The results showed that search efficiency in the gap condition matched that in the single-feature condition and was significantly better than that in the conjunction condition. Thus when the green distractors were presented before the blue items, search became as efficient as it would have been if only the blue items had been presented alone. Attentional prioritization was given to the new blue items, and the old green items were effectively filtered. Interestingly, this gap effect was eliminated if a new dynamic property was introduced into the old green items-for instance, if the old green items had segments removed or added to them as the new blue items appeared. Search in the gap condition did not differ from that in the standard color--form conjunction condition. In this case, the new dynamic property introduced into the old objects was sufficient to overrule the prioritization of attention to new objects. Attention was not automatically captured by the new objects.

\section{Visual Marking}

Watson and Humphreys (1997) went on to explore the conditions under which attentional prioritization to the new items occurred in their search tasks. Most tellingly, they found that prioritization was reduced if subjects had to carry out a resource-demanding secondary task during the period when the old distractor items were displayed.
For example, during the 1,000-msec interval when the old items were displayed, subjects had to identify a series of briefly presented, changing numbers. Under these conditions, the slopes on the search functions in the gap condition were increased relative to the single-feature baseline. Such a result suggests that selection was prioritized to the new items (to produce the gap effect) at least in part by attention being paid to the old items, to deprioritize their selection when the new items appeared. Also, in the dual-task condition there was no evidence of any discontinuities in the slopes of the function in the gap condition as the number of distractors present increased. Such a discontinuity would indicate that resources were applied independently in an all-or-none fashion to each item to be ignored. A reduction in resources would then reduce the total number of items that could be ignored. In contrast, a lack of such a discontinuity is consistent with attentional resources being allocated in parallel to the old distractors, with there being a proportional decrease in this allocation under the dual-task conditions.

Watson and Humphreys (1997) proposed that a process termed visual marking was responsible for the gap effect. The old items in the display were marked by temporary templates set up by the goal state; for static displays, the templates mediated inhibition of the locations of the old items. However, when a secondary task was introduced, the resources allocated to the goal state of inhibiting old distractors were subject to competition, and inhibition was reduced. According to Watson and Humphreys, prioritized selection of new objects can involve deprioritizing attention to old objects by top-down inhibition (marking).

The evidence marshaled in favor of inhibitory visual marking was based on the effective filtering out of old distractors from visual search displays. Inhibition was inferred from the lack of an effect of the distractors on search performance (see also Watson \& Humphreys, 1998). In the present paper, we document new evidence that directly demonstrates that inhibitory processes are involved in marking. In the present experiments, we used a probe-dot procedure (akin to that first pioneered by Klein, 1988) in which, in addition to searching for a target letter on a majority of trials, subjects sometimes had to detect the presence (or absence) of a briefly illuminated dot. As in the standard gap condition used to demonstrate visual marking (Watson \& Humphreys, 1997, 1998), old distractors of one color (green $\mathrm{Hs}$ ) were presented for $1,000 \mathrm{msec}$ before the onset of a second set of items (differing in color), and the target was formed from a conjunction of the old forms and the new color (a blue $\mathrm{H}$ among blue A distractors). The probe dot, when present, could appear at the location of an old distractor or at the location of a new item. The probe-dot trials were accompanied by a brief tone, which signaled that a probe dot rather than a letter detection response had to be made on that trial. We assessed whether detection of the probe dot was worse when it appeared at the location of an old as opposed to a new distractor, as would be expected if there were top-down inhibition of the locations of the old dis- 
a) Conjunction Condition - Search trial (76\%)

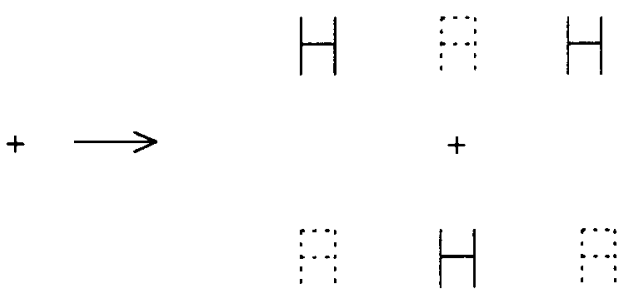

b) Conjunction Condition - Probe trial (24\%)

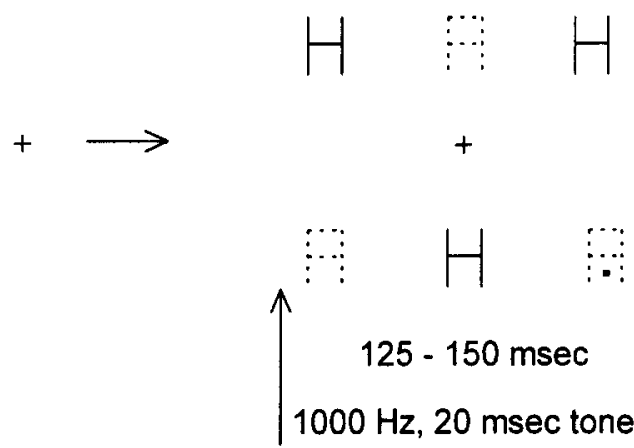

Figure 1. The sequence of displays in a trial from the conjunction condition of Experiment 1 for search trials (a) and probe trials (b).

tractors. As a control, a standard conjunction condition was also included, in which the blue and green distractors appeared simultaneously. In this condition, detection of the probe dot should be unaffected by the color of the distractor where it appeared.

We report three experiments. In Experiments 1 and 3, subjects performed in the gap and the standard conjunction search conditions. In Experiment 2, subjects no longer undertook a search task but rather performed a probedot detection task on each trial. The temporal relations between the old and new distractors were the same as in the gap condition of Experiments 1 and 3 . We expected that in Experiment 2, there would be no incentive to subjects to mark the locations of the old distractors. In this case, any difference in detecting the probe at the locations of old and new stimuli would be reduced.

\section{EXPERIMENT 1}

\section{Method}

\section{Subjects}

Five subjects participated in the study: 3 males and 2 females $19-51$ years of age ( $M=27.2$ years). All were naive with respect to the purpose of the experiment and had self-reported normal or corrected-to-normal vision.

\section{Stimuli and Apparatus}

All displays were generated and presented on a PC-compatible SX-25 based computer driving a VGA graphics card attached to a super VGA monitor. The computer also recorded all subject responses. All stimulus presentations were synchronized with the screen retrace, stimuli were presented against the black background of the computer monitor, and the experiment was performed in a dark room. The viewing distance was $60 \mathrm{~cm}$, although no mechanical means were used to restrict head movements. There were two main conditions: a conjunction condition and a gap condition.

Conjunction condition. Each trial in the conjunction condition began with a blank screen for $1,000 \mathrm{msec}$, followed by a central fixation cross $\left(4 \times 4 \mathrm{~mm}\left[0.38^{\circ} \times 0.38^{\circ}\right]\right)$ for $1,000 \mathrm{msec}$. On $76 \%$ of the trials (search trials), three green (6-bit $\mathrm{RGB}$ values $=3,52,34$ ) and three blue ( $\mathrm{RGB}=17,41,44)$ stimuli were then displayed in two rows of three items to form a rectangle (outer dimensions $42 \mathrm{~mm}$ wide $\times 50 \mathrm{~mm}$ high $\left.\left[4.01^{\circ} \times 4.77^{\circ}\right]\right)$. The top row always consisted of a center blue item flanked by two green items, and the bottom row, a green item flanked by two blue items (the display size was thus always six items). The green items were always $\mathrm{Hs}$ and the blue items As, except on target-present trials, when one of the blue As (chosen randomly) was a blue $\mathrm{H}$. The individual stimuli were $4 \mathrm{~mm}$ wide $\times 8 \mathrm{~mm}$ high $\left(0.38^{\circ} \times 0.76^{\circ}\right)$. The stimuli were presented for 125-150 msec, after which they disappeared, leaving the fixation cross. The fixation cross remained until subjects indicated the presence or absence of the blue $\mathrm{H}$ target by pressing keys $\mathrm{Z}$ or $\mathrm{M}$ on the computer keyboard, after which the next trial began (see Figure 1). The target was present on $50 \%$ of the trials.

On the remaining $24 \%$ of the trials (the probe trials), the search display was accompanied by a $1000-\mathrm{Hz}$ tone of $20-\mathrm{msec}$ duration. This indicated that, rather than search for a blue $\mathrm{H}$ target, subjects should indicate whether a small dim gray probe dot $(0.5 \mathrm{~mm}$ wide $X$ $1 \mathrm{~mm}$ high $\left.\left[0.05^{\circ} \times 0.10^{\circ}\right]\right)$ was present at one of the stimulus items. The probe dot was present on $50 \%$ of the probe trials, and it appeared equally often at the location of a blue or green item. The probe, when present, appeared simultaneously with the search stimuli (as well as the tone that indicated a probe trial), and the probe was positioned in the lower half of an $\mathrm{H}$ or A stimulus (see Figures 1 and 2). After responding to the presence/absence of the probe, the subjects were then prompted to enter a number from 1 to 4 to indicate how confident they were of the response they had given, with 1 indicating least confident and 4 indicating most confident.

Gap condition. The gap condition was the same as the conjunction condition, except that after the fixation cross, the green $\mathrm{H}$ distractors appeared for 1,000 msec alone, after which the blue items (which contained the blue $\mathrm{H}$ target when present) were added to the display. On probe trials, the probe dot (when present) appeared simultaneously with the blue items (and $1000-\mathrm{Hz}$ tone), and again it could appear at the location of either a green or a blue item equally often (see Figure 2).

\section{Design and Procedure}

In the first session, the probe dot luminance and the duration of the displays were manipulated individually for each subject until performance for detecting the probe dot when it fell on a blue item was $80 \%-90 \%$. Establishing this criterion took typically from 2 to 6 blocks of trials. The luminance of the probe dot was varied by changing its color palette RGB values (6-bit resolution). After this, each subject completed 12 blocks of trials ( 6 for each of the conjunction and gap conditions) over a number of sessions. Each block contained a total of 100 trials ( 24 probe trials and 76 search trials), and block order alternated between the two conditions. A single session typically contained 2 blocks of each condition. Whenever possible, separate sessions were run on different days, and the data for each subject were collected within a period of 1 week.

\section{Results}

\section{Search Trials}

The results from the search task trials are shown in Table 1. The mean percentage correct responses were en- 
a) Gap Condition - Search trial ( $76 \%)$

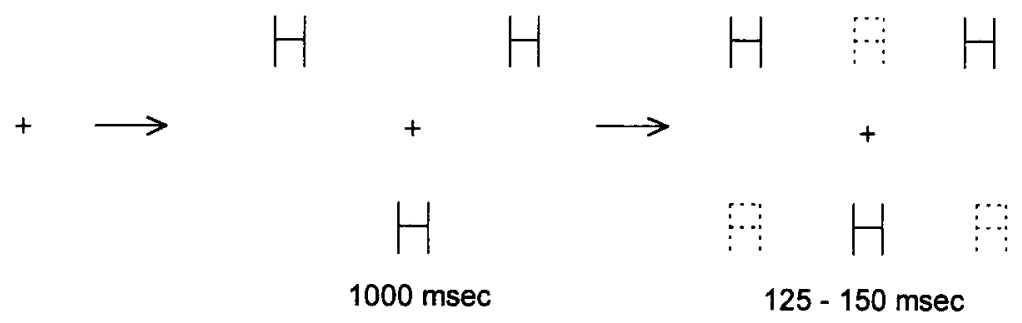

b) Gap Condition - Probe trial (24\%)

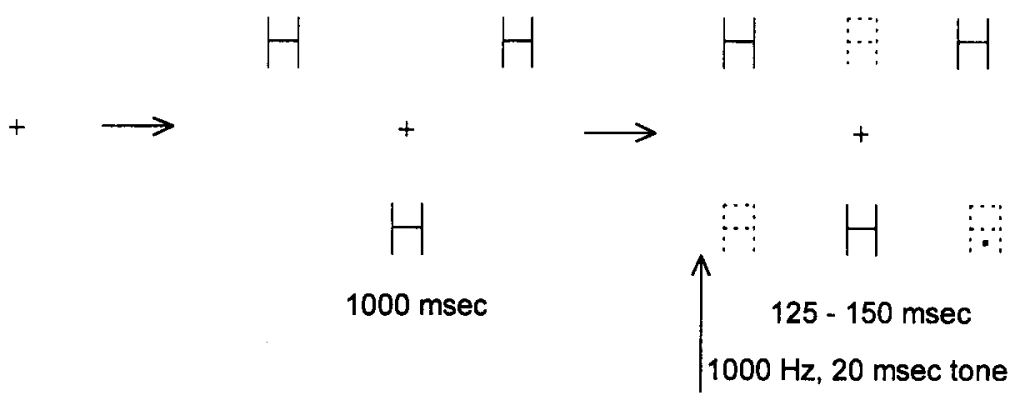

Figure 2. The sequence of displays in a trial from the gap condition of Experiment 1 for search trials (a) and probe trials (b).

tered into a three-way within-subjects analysis of variance (ANOVA) with condition (conjunction or gap), target (absent or present), and block as the main factors. This revealed a significant main effect of condition $[F(1,4)=$ $\left.28.21, M S_{\mathrm{e}}=7.11, p<.01\right]$, with fewer errors made in the gap condition than in the conjunction condition. No other main effects or interactions were significant: for target, $F(1,4)=0.00, M S_{\mathrm{e}}=44.87, p=.974$; block, $F(5,20)=$ $2.28, M S_{\mathrm{e}}=16.02, p=.086$; target $\times$ condition, $F(1,4)=$ $1.62, M S_{\mathrm{e}}=4.29, p=.272$; target $\times$ block, $F(5,20)=2.35$, $M S_{\mathrm{e}}=12.84, p=.078$; condition $\times$ block, $F(5,20)=$ $0.68, M S_{\mathrm{e}}=13.36, p=.641$; target $\times$ condition $\times$ block, $F(5,20)=0.57, M S_{\mathrm{e}}=11.8, p=.722$.

\section{Probe Trials}

Figure 3 and Table 2 show the percentage correct detection of probe dots as a function of probe location and condition. Table 2 also shows the mean confidence values that subjects reported for their responses. We were interested in the detectability of probes falling on green versus blue items in the gap condition (where marking of the green items should have taken place) as compared with the conjunction condition (where marking should not have occurred). We therefore analyzed probe trials on which the probe was present separately from those on which the probe was absent.

\section{Probe-Present Trials}

Probe detection. The mean percentage of correct responses was determined for each block of trials for each subject, and these means were entered into a three-way within-subjects ANOVA. The main factors were probe location (green item or blue item), condition (conjunction or gap), and block.

This revealed a significant main effect of both probe location $\left[F(1,4)=25.55, M S_{\mathrm{e}}=1,056.6, p<.01\right]$ and condition $\left[F(1,4)=75.66, M S_{\mathrm{e}}=89.2, p=.001\right]$. Overall there were more errors in the gap condition and more errors when the probe appeared at the location of a green item. The main effect of block did not reach significance $\left[F(5,20)=0.49, M S_{\mathrm{e}}=392.9, p=.78\right]$. There was also a significant probe $\times$ condition interaction $[F(1,4)=24.37$, $\left.M S_{\mathrm{e}}=237.3, p<.01\right]$. As is shown in Figure 3, in the conjunction condition the detection of a probe falling on a green item was somewhat less accurate than the detection of a probe on a blue item (a 16\% decrease). In contrast, in the gap condition, detection of a probe dot on a green item was considerably more difficult than detection of a probe falling on a blue item (a $44 \%$ decrease). No other interactions approached significance: for probe $\times$ block, $F(5,20)=0.80, M S_{\mathrm{e}}=279.0, p=.566$; condition

Table 1

Mean Percentage of Correct Responses on

Search Trials, as a Function of Condition and Target Absence/Presence in Experiment 1

\begin{tabular}{lcc}
\hline & \multicolumn{2}{c}{ Target } \\
\cline { 2 - 3 } Condition & Absent & Present \\
\hline Conjunction & 95.1 & 95.6 \\
Gap & 98.2 & 97.7 \\
\hline
\end{tabular}




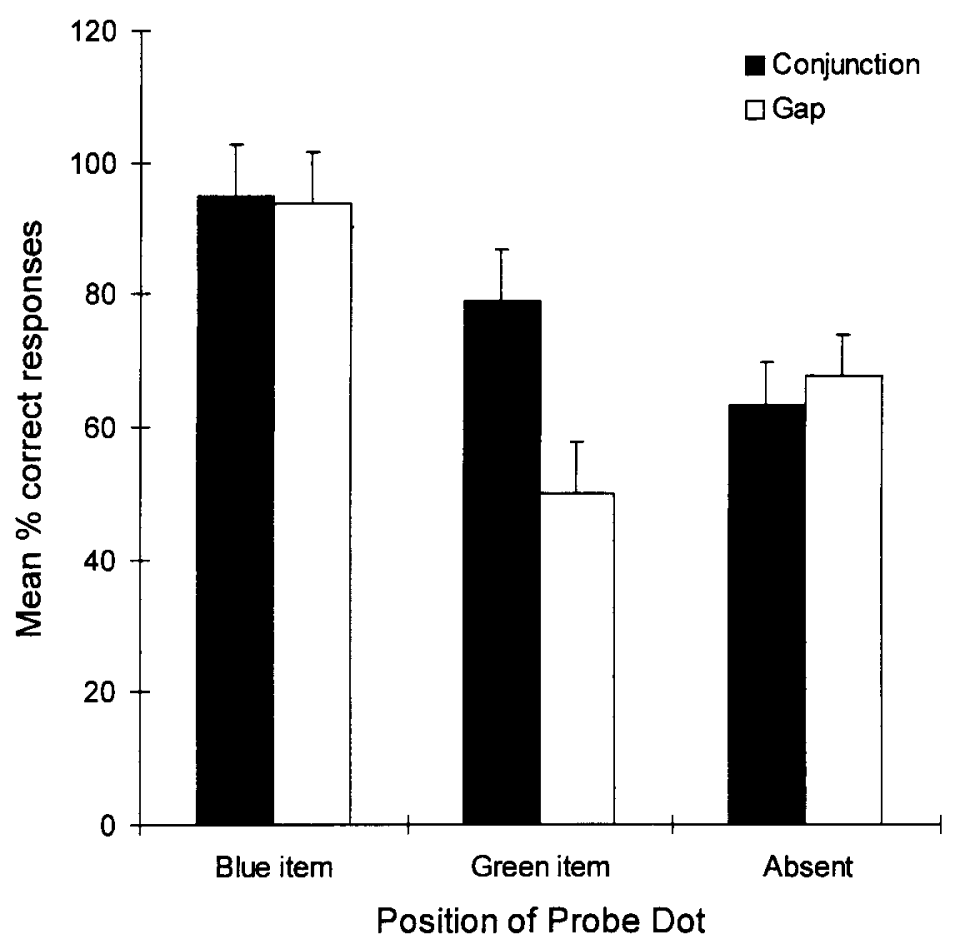

Figure 3. Mean percentage of correct responses for Experiment 1, as a function of condition (conjunction or gap) and probe-dot position (blue item, green item, or absent). Error bars show $\pm 95 \%$ confidence intervals appropriate to a within-subjects analysis (see Loftus \& Masson, 1994).

$\times$ block, $F(5,20)=0.25, M S_{\mathrm{e}}=286.3, p=.934$; probe $\times$ condition $\times$ block, $F(5,20)=0.48, M S_{\mathrm{e}}=226.2, p=.785$.

Confidence judgments. The mean confidence judgments for correct probe trials only are shown in Table 2; they were analyzed with the same procedure as that above. Overall, the confidence judgments closely mirrored the accuracy responses, with greater accuracy corresponding to greater confidence. There were significant main effects of probe location $\left[F(1,4)=13.98, M S_{\mathrm{e}}=2.10, p<.05\right]$ and condition $\left[F(1,4)=10.46, M S_{\mathrm{e}}=0.135, p<.05\right]$. Overall, subjects were more confident of their responses when the probe fell on a blue item than when it fell on a green item and were more confident in the conjunction condition. There was also a significant probe location $\times$ condition interaction $\left[F(1,4)=32.67, M S_{\mathrm{e}}=0.098, p=.005\right]$. In the conjunction condition, confidence was similar for probes falling on both green and blue items. In contrast, in the gap condition, confidence was much lower when the probe fell on a green item than when it fell on a blue item. No other main effects or interactions were significant: for block, $F(5,20)=1.19, M S_{\mathrm{e}}=0.23, p=.35$; probe $\times$ block, $F(5,20)=2.28, M S_{\mathrm{e}}=0.167, p=.085$; condition $\times$ block, $F(5,20)=0.78, M S_{\mathrm{e}}=0.30, p=.578$; probe $\times$ condition $\times$ block, $F(5,20)=1.08, M S_{\mathrm{e}}=0.201, p=.402$.

\section{Probe-Absent Trials}

Probe detection. There was a significant main effect of block $\left[F(5,20)=8.23, M S_{\mathrm{e}}=173.5, p<.001\right]$; accuracy increased slightly over the first two blocks. However, neither the main effect of condition $\left[F(1,4)=1.91, M S_{\mathrm{e}}=154.7\right.$, $p=.239]$ nor the condition $\times$ block interaction $[F(5,20)=$ $\left.2.05, M S_{\mathrm{e}}=158.1, p=.114\right]$ approached significance.

Confidence judgments. No main effects or interactions proved significant: for condition, $F(1,4)=0.95$, $M S_{\mathrm{e}}=0.24, p=.385 ;$ block, $F(5,20)=0.55, M S_{\mathrm{e}}=0.189$, $p=.737$; condition $\times$ block, $F(5,20)=0.29, M S_{\mathrm{e}}=$ $0.083, p=.915$.

\section{Discussion}

Several points should be made with regard to the results of Experiment 1. On search trials (in which the subjects searched for the blue $\mathrm{H}$ target), performance was better in the gap condition than in the conjunction condition. Thus, presenting the green distractors before the blue (allowing them to be marked) produced a selection advantage for the blue items. This advantage for the new items in the gap condition is consistent with the basic gap effect found previously using RT measures in search tasks (Watson \&

Table 2

Mean Percentage of Correct Responses and Decision Confidence for the Detection of a Probe Dot, as a Function of Condition and Probe Position in Experiment 1

\begin{tabular}{|c|c|c|c|c|c|c|}
\hline \multirow[b]{3}{*}{ Condition } & \multicolumn{6}{|c|}{ Position of Probe Dot } \\
\hline & \multicolumn{2}{|c|}{ Blue Item } & \multicolumn{2}{|c|}{ Green Item } & \multicolumn{2}{|c|}{ Absent } \\
\hline & $\%$ Corr. & Conf. & $\%$ Corr. & Conf. & $\%$ Corr. & Conf \\
\hline Conjunction & 95.0 & 3.7 & 78.9 & 3.1 & 63.3 & 2.3 \\
\hline Gap & 93.9 & 3.8 & 50.0 & 2.5 & 67.8 & 2.4 \\
\hline
\end{tabular}


Humphreys, 1997, 1998), and it extends the findings on visual marking to the use of briefly presented displays. ${ }^{1}$

However, of most interest was the performance on probe trials. Here the results showed that in the conjunction condition, probe detection was generally accurate and there was relatively little difference in the detection of probes falling on green or blue items. Performance was somewhat worse when the dot fell at the location of green distractors (a 16\% decrease), presumably because it was then less discriminable (either because of the color or because of the shape of the old relative to the new distractors). However, in the gap condition, where it was expected that visual marking would be applied to the old distractors, probes were considerably harder to detect when they fell at the location of a green (marked) item relative to a blue (nonmarked) item (a 44\% decrease). ${ }^{2}$ In addition, even when subjects correctly detected the occurrence of a probe dot on a green marked item, they were still much less confident of their response than they were when the probe fell on a blue item (or on a green item in the conjunction condition).

These last findings are consistent with visual marking operating via the application of inhibition to the locations of old, to-be-ignored visual stimuli. Thus visual marking has been demonstrated with a different paradigm from that in previous studies and has been shown to be inhibitory in nature (relative to the conjunction baseline). However, alternative interpretations of the data remained possible. For instance, the old green distractors might have acted as forward masks on the probes, reducing their detectability at old locations. It was also possible that the new blue items acted as peripheral cues summoning attention to their locations automatically and perhaps away from the locations of the green items (Jonides, 1981; Posner, Snyder, \& Davidson, 1980). In Experiment 2, we tested these possibilities.

In Experiment 2, subjects performed only in the gap condition and all the trials were now probe trials in which the subjects had to detect the presence or absence of a probe dot; there were no search trials. Other than this, the displays were identical to those in the gap condition of Experiment 1. Since all the trials were probe trials, there was now no incentive for subjects to mark the initial old green distractors when they were presented alone; there were no search trials for which marking would provide a search advantage. If visual marking was not applied to the old items, probe detection would now not differ between the green and blue stimuli. However, if forward masking or peripheral cue effects were responsible for the differences in probe detection in Experiment 1, the same difference should be present here.

\section{EXPERIMENT 2}

\section{Method}

\section{Subjects}

The subjects from Experiment 1 participated in Experiment 2.

\section{Design and Procedure}

The design was essentially the same as that of Experiment 1, except that only the gap condition was presented and each block of tri- als consisted only of probe trials. Each subject completed eight blocks of trials within one to three sessions, with a total of 48 trials per block. The stimulus duration and brightness of the probe dots used in Experiment 1 were used again in Experiment 2 for each subject.

\section{Results}

The mean percentages of correct responses for the detection of the probes are shown in Figure 4, and the subjects' confidence in those responses is shown in Table 3.

\section{Probe Detection}

A two-way within-subjects ANOVA with probe (blue item, green item, or absent) and block revealed no significant main effects or interactions of probe $[F(2,8)=1.45$, $\left.M S_{\mathrm{e}}=877.0, p=.290\right]$, block $\left[F(7,28)=0.87, M S_{\mathrm{e}}=\right.$ $132.9, p=.544]$, or probe $\times$ block $[F(14,56)=0.70$, $\left.M S_{\mathrm{e}}=151.2, p=.765\right]$.

\section{Confidence Ratings}

There was a significant main effect of probe $[F(2,8)=$ $\left.25.99, M S_{\mathrm{e}}=0.53, p<.001\right]$; subjects were less confident of their responses when the probe dot was absent. Neither the main effect of block $\left[F(7,28)=1.15, M S_{\mathrm{e}}=0.07\right.$, $p=.363]$ nor the probe $\times$ block interaction $[F(14,56)=$ $\left.0.74, M S_{\mathrm{e}}=0.09, p=.729\right]$ proved significant.

\section{Comparison With Experiment 1}

Probe detection accuracy on probe-target-present trials in the gap condition was compared across Experiments 1 and 2 using a two-way within-subjects ANOVA with probe position (blue or green item) and experiment as the main factors. This revealed a significant main effect of probe position $\left[F(1,4)=36.42, M S_{\mathrm{e}}=89.16, p<.005\right]$ and a significant probe position $\times$ experiment interaction $\left[F(1,4)=19.94, M S_{\mathrm{e}}=84.88, p=.011\right]$. In Experiment 2, in which there was no advantage for subjects to visually mark the old items, there was little difference between accuracy for a probe falling on a blue (new) as opposed to a green (old) item. In contrast, in Experiment 1, in which visual marking was advantageous, detection of a probe dot at the location of a green (old) item was much less accurate than the detection of a probe dot falling at the location of a blue (new) item.

\section{Discussion}

The results showed that when there was no incentive for subjects to mark the initial old items, probe detection at the location of an old item was as easy as probe detection at the location of a new item. This should not have been the case if forward masking or peripheral cuing effects were the cause of the difference in probe detection in the gap condition in Experiment 1. The inhibition in the probe-dot detection task was contingent on subjects' marking the locations of old distractors. This result provided further evidence that visual marking is a top-down flexible process that can be applied or not applied, depending on current task demands and behavior (cf. Watson \& Humphreys, 1997, 1998); marking did not occur 


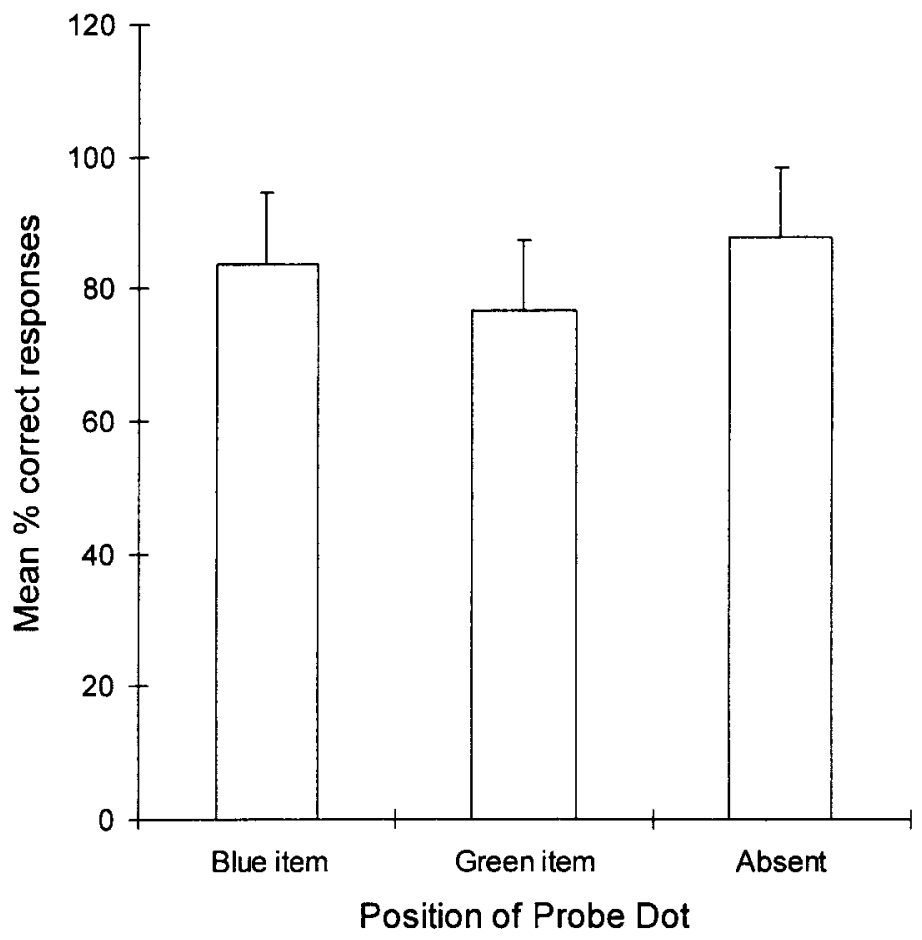

Figure 4. Mean percentage of correct responses for Experiment 2, as a function of probe-dot position (blue item, green item, or absent). Error bars show $\pm 95 \%$ confidence intervals appropriate to a within-subjects analysis (Loftus \& Masson, 1994).

automatically, when subjects did not have to search letter displays.

\section{EXPERIMENT 3}

In Experiment 1, the six display items always fell in the same configuration and positions across all trials. This display method was chosen so that the locations of the blue (potential target) stimuli would have no relative advantage in the gap condition. For instance, we could have varied the positions of the stimuli so that the blue and green items swapped positions randomly across trials. This would have had the effect that in the conjunction condition, just before the display, subjects would have had no idea about the likely position of the blue (target) items. However, in the gap condition, the positions of the initial green items would have indirectly signaled the positions of the forthcoming blue (target) items (i.e., they would appear in the remaining three positions). As it was, with a fixed configuration, subjects always knew the positions of the blue items in both the gap and the conjunction conditions, removing this potential confound. However, it was possible that subjects might have developed an endogenous template for this fixed display configuration that allowed them to prioritize or bias the three locations that contained the blue items. Indeed, previous studies have shown that multiple locations can be tagged for attentional priority (Wright, 1994; see also Pylyshyn, 1989, Pylyshyn et al., 1994, Yantis \& Johnson, 1990, Yantis \&
Jones, 1991). Such an account might explain the results of Experiment 1 in terms of the application of attention to target locations at the expense of distractor locations, rather than the direct inhibition of distractor locations. However, note that it might not be possible to tag multiple empty locations for priority (as would have been required here) unless they are occupied (Pylyshyn et al., 1994). Indeed, Watson and Humphreys (1997) tested and ruled out an account of visual marking based on the spatial cuing of likely target regions by presentation of the first set of distractors in the gap condition. In their Experiment 6, a set of green blocks was presented prior to a subsequent set of letters that had to be searched. The green blocks could serve as spatial precues (similar to the old letters in a standard gap search procedure), facilitating target detection. However, Watson and Humphreys (1997) found no effect of such precues on search. In addition, the template account itself encounters difficulties when applied to the present results. For instance, the account would have to be expanded to explain why the detection of a probe dot on

Table 3

Mean Percentage of Correct Responses and

Decision Confidence for the Detection of a Probe Dot, as a Function of Probe Position for Experiment 2

\begin{tabular}{|c|c|c|c|c|c|}
\hline \multicolumn{6}{|c|}{ Position of Probe Dot } \\
\hline \multicolumn{2}{|c|}{ Blue Item } & \multicolumn{2}{|c|}{ Green Item } & \multicolumn{2}{|c|}{ Absent } \\
\hline$\%$ Corr. & Conf. & $\%$ Corr. & Conf. & $\%$ Corr. & Conf. \\
\hline 83.8 & 3.6 & 76.7 & 3.7 & 87.8 & 2.6 \\
\hline
\end{tabular}




\begin{tabular}{|l|l|l|l|l|l|l|l|l|l|}
\hline & & & & & & & & & \\
\hline & 3 & 3 & 3 & 3 & 3 & 3 & 3 & 3 & \\
\hline & 3 & 2 & 2 & 2 & 2 & 2 & 2 & 3 & \\
\hline & 3 & 2 & 1 & 1 & 1 & 1 & 2 & 3 & \\
\hline & 3 & 2 & 1 & & & 1 & 2 & 3 & \\
\hline & 3 & 2 & 1 & & & 1 & 2 & 3 & \\
\hline & 3 & 2 & 1 & 1 & 1 & 1 & 2 & 3 & \\
\hline & 3 & 2 & 2 & 2 & 2 & 2 & 2 & 3 & \\
\hline & 3 & 3 & 3 & 3 & 3 & 3 & 3 & 3 & \\
\hline & & & & & & & & & \\
\hline
\end{tabular}

Figure 5. The stimulus matrix and the definition of the three radial zones (labeled $1,2,3$ ) into which the probe dot could fall when present. The matrix lines were not visible to the subject.

a green item was much less accurate in the gap condition than in the conjunction condition in the search experiment (Experiment 1). To explain this, one would have to argue that the appearance of the green items alone somehow enabled the endogenous template to be more effective, perhaps by providing a memory cue for the display configuration.

Despite these objections, in order to test the template account directly and the flexibility of visual marking further, we conducted Experiment 3. In this experiment rather than having a fixed configuration for the display elements, we placed them randomly within the cells of a $10 \times 10$ virtual matrix on every trial. This removed the possibility that subjects might have developed an endogenous template for the blue item locations, because (1) the locations of the new (blue) items were not consistent or predictable in advance and (2) across trials, any particular location was equally likely to be occupied by either a blue or a green item. In order to control for possible different retinal eccentricities of the probe dot, we defined three zones from fixation into which the probe dot, when present, could fall. We also increased the total display size to eight items. This display size was the "middle" display size typically used in previous visual marking experiments (Watson \& Humphreys, 1997, 1998).

\section{Method}

\section{Subjects}

Three subjects participated: 1 male and 2 females between 21 and 27 years of age ( $M=23.7$ years). All were naive with respect to the purpose of the experiment, had self-reported normal or corrected-to-normal vision, and were paid for their participation according to the number of blocks completed.

\section{Stimuli and Apparatus}

The stimuli were essentially the same as in Experiments 1 and 2, with the following exceptions. All displays were generated and presented on a Pentium-based PC-compatible computer running at $120 \mathrm{MHz}$ driving an SVGA graphics card attached to an SVGA monitor. The conjunction and gap conditions were the same as in Experiment 1, except that the total display size was eight items and the items were arranged randomly on each trial within the cells of a $10 \times 10$ invisible matrix, which measured $102 \mathrm{~mm}$ horizontally $\times 120 \mathrm{~mm}$ vertically $\left(9.72^{\circ} \times 11.42^{\circ}\right)$.

As in Experiment 1 , in the conjunction condition all the items appeared simultaneously and in the gap condition the green items preceded the blue items by $1,000 \mathrm{msec}$. Within a block of 100 trials, 24 were probe trials and 76 were search trials. Out of the 24 probe trials, there were 12 probe-dot-present trials and 12 probedot-absent trials. When the probe dot was present, it was constrained to fall within one of three concentric zones. These square zones were defined as two, three, or four matrix squares from fixation. These equated to nominal distances of 20,32 , and $44 \mathrm{~mm}$ $\left(1.91^{\circ}, 3.06^{\circ}, 4.20^{\circ}\right)$ from fixation (see Figure 5).

On a probe-dot-present trial, the probe dot was positioned so that it fell into one of the cells defined by its specified zone. The appropriate display item (green or blue) was then positioned at the probe location. The particular cell of the specified zone into which the probe fell was chosen at random. In this way, the average retinal eccentricity of a probe dot was constant across conditions and blocks. The remaining display items were then positioned. Following a probe trial, subjects were not prompted to indicate their decision confidence.

\section{Design and Procedure}

In the initial session, the luminance of the probe dot was varied for each subject until their performance reached $80 \%-90 \%$ correct on probe-present trials in the conjunction condition. It typically took from two to four blocks of trials to establish this criterion. This luminance value was then used for all the remaining conditions. The duration of the display was fixed at $414.7 \mathrm{msec}$ for all subjects. As expected, a longer display duration proved necessary than in Experiment 1 because of the increased number of display elements and the random configurations presented.

After determining the luminance of the probe dot, each subject performed a total of 12 blocks of trials, 6 of the gap condition and 6 of the conjunction condition. Each subject received 2 blocks of each condition within a single session. All sessions were run on different days. Different sessions for each subject were run either on consecutive days or with a single day between sessions.

\section{Results}

\section{Search Trials}

The results for the search trials are shown in Table 4. The mean percentages of correct responses were analyzed as in Experiment 1. Although there was a trend for more accurate performance in the gap condition than in the conjunction condition, no main effects or interactions proved statistically significant. This was most likely due to performance's being close to ceiling as a result of the relatively long display duration that was used.

\section{Probe Trials}

Figure 6 shows the percentages of correct detection of probe dots as a function of probe location and condition. 
Table 4

Mean Percentage of Correct Responses on

Search Trials, as a Function of Condition

and Target Absence/Presence in Experiment 3

\begin{tabular}{lcc}
\hline & \multicolumn{2}{c}{ Target } \\
\cline { 2 - 3 } Condition & Absent & Present \\
\hline Conjunction & 92.0 & 87.9 \\
Gap & 93.4 & 92.1 \\
\hline
\end{tabular}

As in Experiment 1, our main interest was in the detectability of probes falling on green versus blue items in the gap condition as compared with the conjunction condition. We thus analyzed probe-present trials separately from probe-absent trials.

\section{Probe-Present Trials}

The data were analyzed in the same way as in Experiment 1 . This analysis revealed a significant main effect of probe position $\left[F(1,2)=31.08, M S_{\mathrm{e}}=432.1, p<.05\right]$. Overall, probes were more accurately detected when they were at the location of a blue item as opposed to a green item. The main effect of condition also approached significance $\left[F(1,2)=12.25, M S_{\mathrm{e}}=138.9, p=.073\right]$; probes tended to be more accurately detected in the conjunction condition than in the gap condition. Of most interest was a significant probe position $\times$ condition inter- action $\left[F(1,2)=60.75, M S_{\mathrm{e}}=46.3, p=.016\right]$. The difference in probe detection accuracy for blue-item versus green-item probes was much greater in the gap condition (a 39.8\% decrease) than in the conjunction condition (a $14.8 \%$ decrease). No other main effect or interaction was significant.

\section{Probe-Absent Trials}

No main effects or interaction proved significant.

\section{Eccentricity of Probe Dots}

The effect of probe-dot eccentricity on probe-present trials was examined in a three-way within-subjects ANOVA with the main factors of probe position (blue/ green), condition (gap/conjunction), and eccentricity (Zone 1,2 , or 3 ). This revealed a significant main effect of probe eccentricity $\left[F(2,4)=11.34, M S_{\mathrm{e}}=233.41, p<\right.$ $.05]$. As might be expected from visual acuity considerations, accuracy decreased as the distance from fixation of the probe dot increased. Interestingly, however, eccentricity did not interact significantly with any other factors. This suggests that visual marking had similar effects on old objects across the visual field.

\section{Discussion}

Experiment 3 replicated Experiment 1, but with displays in which the positions of the blue and the green

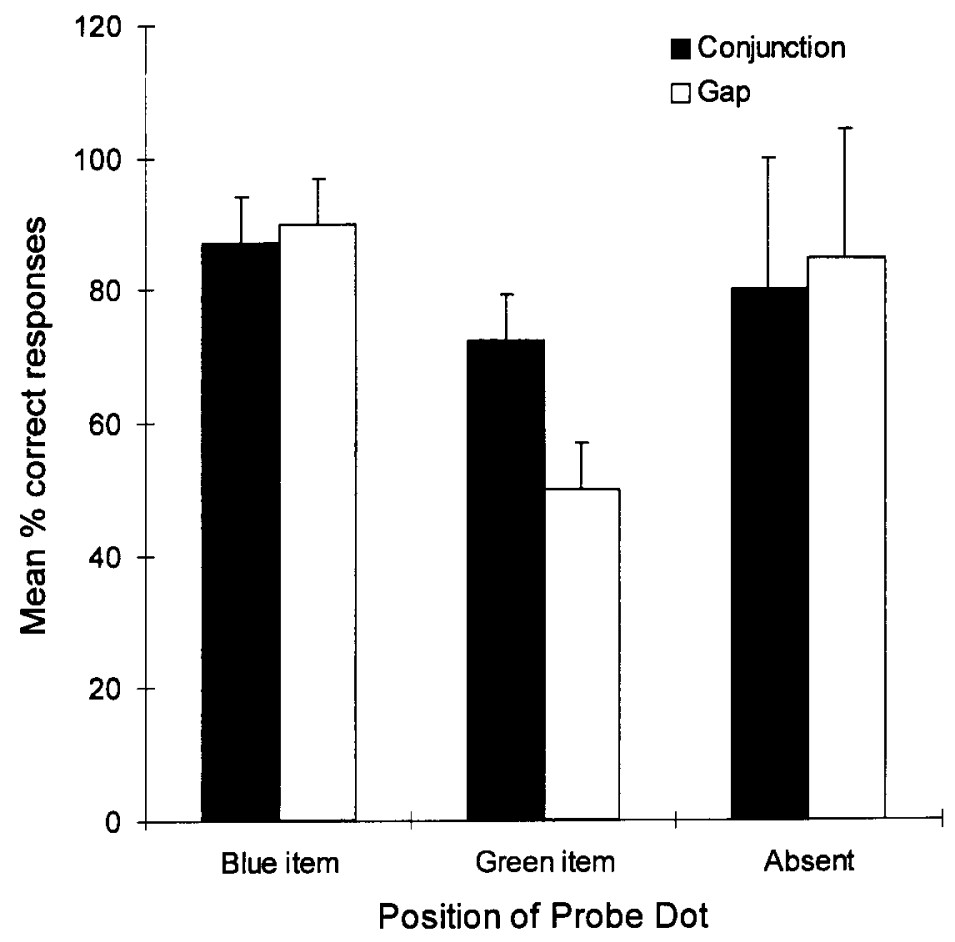

Figure 6. Mean percentage of correct responses for Experiment 3, as a function of condition (conjunction or gap) and probe-dot position (blue item, green item, or absent). Error bars show $\pm 95 \%$ confidence intervals appropriate to a within-subjects analysis (Loftus \& Masson, 1994). 
items were random on every trial and so precluded the development and use of an endogenous template to facilitate processing of potential target stimuli. In addition, the total number of display items was increased to eight to match previous visual marking experiments more closely. Despite these changes, essentially the same pattern of results was obtained. Namely, the detection accuracy for a probe dot on a green item was much higher when all the items appeared simultaneously (in the conjunction condition) than when the green items appeared before the blue (in the gap condition) and could thus be visually marked. Interestingly, the difference between probe detection accuracy on a blue versus a green item in Experiment 3 was quite close to the results obtained in Experiment 1: conjunction condition, Experiment $1=16 \%$ decrement, Experiment $3=15 \%$ decrement; gap condition, Experiment $1=44 \%$ decrement, Experiment $3=40 \%$ decrement. The results show that the decrement for the detection of a probe dot on a green item in the gap condition of Experiment 1 was not contingent on subjects' being able to develop an endogenous template of the stimulus configuration through being able predict the locations of the stimuli.

The data from this experiment also allowed an initial inspection of how visual marking may operate across the visual field. The results suggest that visual marking was equally effective across differing visual field eccentricities. This is consistent with visual marking's being based on the parallel application of inhibition to old locations across the whole visual field (at least within the range of eccentricities that were measured here).

\section{GENERAL DISCUSSION}

In this research, we investigated the phenomenon of visual marking using a probe-dot-detection procedure. Experiment 1 showed that in a color-form conjunction task the effect of presenting one set of distractors before the remaining items was to make search more efficient for the second set of items, relative to when all the items were presented simultaneously. This replicates the basic gap effect found by Watson and Humphreys (1997) and extends it to briefly presented displays. However, of most importance was the finding that the detection of a small probe dot at a marked location was greatly impaired in comparison with the detection of the same dot at the location of an unmarked item. The same impairment in detection was not found in a standard conjunction search condition, used here as a baseline. The impairment directly implicates inhibition in the process of visual marking. Experiment 2 ruled out two possible alternative accounts of our findings. These were (1) that the initial distractors acted as forward masks and (2) that the second set of items acted as peripheral cues, automatically attracting attention to their locations. These last accounts can be rejected, because there was no impairment of probedot detection at old locations when subjects never had to search letter displays. The fact that marking did not occur when subjects had no rationale for inhibiting the initial items provides further evidence that visual marking is a top-down process that the observer applies flexibly, depending on current task requirements (cf. Watson \& Humphreys, 1997).

One potential difficulty with Experiment 1 was that the display items always appeared in the same locations across all trials. Therefore, subjects might have built up an endogenous template of the display that helped them prioritize the blue items by applying attention to their locations at the expense of attention to the locations of the green items. This account explains the results in terms of a biased application of attention to target locations rather than the inhibition of distractor locations. However, this account relies on the (unproven) ability of subjects to tag or bias empty locations for attention (see Watson \& Humphreys, 1997, Experiment 6, for contrary evidence) and the account has difficulty in explaining why detection of a probe falling at the location of a green (old) item was much worse in the gap condition than in the conjunction condition. For the account to be tenable, one must argue that the appearance of the green items on their own in the gap condition resulted in a more efficient application of the endogenous template. The template account was tested directly in Experiment 3.

Experiment 3 replicated Experiment 1, but with displays in which the items were randomly placed on each trial. This random placement precluded the possibility of the development of an endogenous template, and furthermore, all locations had an equal probability of containing either a target or a distractor item. Despite this change, there was essentially the same pattern of results as in Experiment 1 . Namely, a probe dot was much more difficult to detect when it fell at the location of an old green (marked) item in the gap condition than when it fell at the location of a green item in the conjunction condition, which did not have the opportunity to be marked in advance of the blue distractors. This supports our account that inhibition via a process of visual marking is applied to the locations of old distractors when it is advantageous for those items to be ignored. Interestingly, evidence from Experiment 3 also suggested that inhibition was applied in parallel to items across the visual field (at least under the present display conditions).

Probably the closest experiments in the current literature to the present experiments are those of Klein (1988) and Cave and Kim and colleagues (Cepeda, Cave, Bichot, \& Kim, 1998; Kim \& Cave, 1995). Klein tested whether during a standard visual search task requiring the application of serial attention there would be inhibition of the positions of previously examined items (perhaps by a process of inhibition of return; Posner \& Cohen, 1984). Such inhibition would bias attention against returning to already rejected stimuli. In Klein's study, following presentation of a conjunction search display, subjects had to detect the presence of a small probe target that could appear at a previously occupied or empty location. The results showed that probe detection was more difficult when it ap- 
peared in a previously occupied location. However, when a single-feature search task was presented (which does not require the serial application of attention), no difference was found for probes falling in a previously empty or occupied location (but see Wolfe \& Pokorny, 1990, for a failure to replicate). Klein suggested that the inhibition of locations that had been previously examined by attention would lead to more efficient search through complex displays. Cave and Kim and collaborators have also used a probe-detection task interpolated between trials on a primary search task. In their procedure, search displays were presented briefly and RTs were measured to probes. They found that RTs were delayed when probes fell at distractor locations relative to when they fell at the locations of targets (or in unfilled locations close to targets). These results suggest that targets are selected at least in part by inhibition of the locations of distractors. We too used a probedot-detection paradigm; however, we were interested in inhibition applied to one set of items that appeared before a second set of stimuli. Our findings showed that old stimuli could be inhibited but only when there was an advantage for subjects to ignore the old stimuli. Inhibition was applied in a goal-directed way to the locations of old items. When there was no advantage to be gained by inhibiting old locations (in the nonsearch condition), inhibition appeared not to be applied. Since performance in the gap condition was measured here relative to that in the conjunction condition, it appears that top-down inhibition to old locations is applied over and above effects operative in simultaneous conjunction search (cf. Kim \& Cave, 1995). Whether these inhibitory processes share a common mechanism is a question awaiting future research.

\section{REFERENCES}

ALLPORT, D. A. (1987). Selection for action: Some behavioral and neurophysiological considerations of attention and action. In H. Heuer \& A. F. Sanders (Eds.), Perspectives on perception and action (pp. $395-$ 419). Hillsdale, NJ: Erlbaum.

Cepeda, N. J., Cave, K. R., Bichot, N. P., \& Kim, M.-S. (1998). Spatial selection via feature-driven inhibition of distractor locations. Perception \& Psychophysics, 60, 727-746.

DunCan, J. (1980). The locus of interference in the perception of simultaneous stimuli. Psychological Review, 87, 272-300.

HuMPHREYS, G. W., \& MÜLLER, H. J. (1993). Search via recursive rejection (SERR): A connectionist model of visual search. Cognitive Psychology, 25, 43-110.

JONIDES, J. (1981). Voluntary versus automatic control over the mind's eye's movement. In J. B. Long \& A. D. Baddeley (Eds.), Attention and Performance $I X$ (pp. 187-203). Hiilsdale, NJ: Eribaum.

KIM, M.-S., \& CAVE, K. R. (1995). Spatial attention in visual search for features and feature conjunctions. Psychological Science, 6, 376-380.

KLEIN, R. (1988). Inhibitory tagging system facilitates visual search. Nature, 334, 430-431.

Loftus, G. R., \& Masson, M. E. J. (1994). Using confidence intervals in within-subject designs. Psychonomic Bulletin \& Review, 1, 476-490.

POSNER, M. I., \& COHEN, Y. (1984). Components of visual orienting. In H. Bouma \& D. G. Bouwhuis (Eds., Attention and performance $X$ : Control of language processes (pp. 531-556). Hillsdale, NJ: Erlbaum.

PosNer, M. I., SNYDER, C. R. R., \& Davidson, B. J. (1980). Attention and the detection of signals. Journal of Experimental Psychology: General, 109, 160-174.
Pylyshyn, Z. W. (1989). The role of location indexes in spatial perception: A sketch of the FINST spatial-index model. Cognition, 32, 65-97.

Pylyshyn, Z. [W.], Burkell, J., Fisher, B., Sears, C., Schmidt, W, \& TRICK, L. (1994). Multiple parallel access in visual attention. Canadian Journal of Experimental Psychology, 48, 260-283.

TOWNSEND, J. T. (1972). Some results on the identifiability of parallel and serial processes. British Journal of Mathematical \& Statistical Psychology, 25, 168-199.

Treisman, A. M., \& Gelade, G. (1980). A feature-integration theory of attention. Cognitive Psychology, 12, 97-136.

Watson, D. G., \& Humphreys, G. W. (1995). Attention capture by contour onsets and offsets: No special role for onsets. Perception \& Psychophysics, 57, 583-597.

Watson, D. G., \& Humphreys, G. W. (1997). Visual marking: Prioritizing selection for new objects by top-down attentional inhibition. Psychological Review, 104, 90-122.

WATSON, D. G., \& Humphreys, G. W. (1998). Visual marking of moving objects: A role for top-down feature-based inhibition in selection. Journal of Experimental Psychology: Human Perception \& Performance, 24, 946-962.

WOLFE, J. M., \& POKORNY, C. W. (1990). Inhibitory tagging in visual search: A failure to replicate. Perception \& Psychophysics, 48, 357362.

WRIGHT, R. D. (1994). Shifts of visual attention to multiple simultaneous location cues. Canadian Journal of Experimental Psychology, 48, 205-217.

YANTIS, S. (1993). Stimulus-driven attentional capture and attentional control settings. Journal of Experimental Psychology: Human Perception \& Performance, 19, 676-681.

YANTIS, S., \& HILLSTROM, A. P. (1994). Stimulus-driven attentional capture: Evidence from equiluminant visual objects. Journal of Experimental Psychology: Human Perception \& Performance, 20, 95-107.

YANTIS, S., \& JOHNSON, D. N. (1990). Mechanisms of attentional priority. Journal of Experimental Psychology: Human Perception \& Performance, 16, 812-825.

YANTIS, S., \& JONES, E. (1991). Mechanisms of attentional selection: Temporally modulated priority tags. Perception \& Psychophysics, $\mathbf{5 0}$, 166-178.

YANTIS, S., \& JonidES, J. (1984). Abrupt visual onsets and selective attention: Evidence from visual search. Journal of Experimental Psychology: Human Perception \& Performance, 10, 601-621.

YANTIS, S., \& JoNIDES, J. (1996). Attentional capture by abrupt visual onsets: New perceptual objects or visual masking? Journal of Experimental Psychology: Human Perception \& Performance, 22, 1505-1513.

\section{NOTES}

1. The difference between the gap and the conjunction condition in the search task is encouraging, although to some degree unexpected given that performance in this task was close to ceiling. Recall that the durations of the displays were manipulated in the first session contingent on performance in the probe-dot-detection task and not on performance in the search task. Optimal display durations required to show maximum differences between the gap and conjunction conditions in the search task would have been much shorter, given the current display parameters.

2 . We note that data from a control study revealed the same pattern of results when the two colors of the stimuli (green/blue) were interchanged. Accuracy on search trials was overall better in the gap condition, and on probe trials, the detection of a probe was impaired when it fell at the location of an old marked item in the gap condition. This precludes the possibility that the particular colors used were responsible for the present findings.

(Manuscript received August 19, 1996; revision accepted for publication October 24, 1998.) 